\title{
New low radiation background material technology
}

\author{
Laura Cardani* \\ Dipartimento di Fisica - Sapienza Università di Roma and INFN - Roma (Italy), and Physics \\ Department, Princeton University (NJ, USA) \\ E-mail: laura.cardanieromal.infn.it
}

\begin{abstract}
More and more experiments need materials with extremely low radioactive content. The requirements on contaminations levels are becoming so strict that "standard" techniques can not reach the sufficient sensitivity to determine if a material complies with the specifications. In this contribution I use two widely applied materials (crystals and copper) as examples to show the main issues in the achievement of a high radio-purity. Than, I present two newly developed technologies to probe bulk and surface contaminations of several materials with unprecedented sensitivity.
\end{abstract}

INFN Workshop on Future Detectors for HL-LHC

16-18 December, 2015

Aula Magna della Cavallerizza Reale, Torino, Italy

${ }^{*}$ Speaker. 


\section{Introduction}

The production of materials with lower and lower radioactive contaminations has always been among the most difficult challenges for several physics sectors. Well known examples are experiments searching for rare events, such as neutrino-less double beta decay, $\alpha$-decays with half-lives exceeding $10^{18}$ years, or dark matter interactions. These experiments need to work with a background as close as possible to zero. For this reason, the detectors are always located in deep underground laboratories and equipped with state-of-the-art shielding and vetoes agains the external radioactivity.

When all the environmental radioactivity is suppressed, the detector itself may become the most dangerous source of background. For this reason, most of the next-generation experiments are performing an intense $R \& D$ activity devoted to the selection of radio-pure materials. In most of the cases, the requirements on the radioactivity level are so stringent, that none of the available screening technologies is able to reach the sufficient sensitivity to establish if the material complies with the needs of the experiment. In this contribution I focus on an active and a passive element used by many projects: crystals and copper. Starting from these examples, I will present some of the most advanced screening technologies, that can be of interest also for many other materials.

\section{Crystals}

Crystals are chosen by experiments in different physics sectors, as they can be used as calorimeters, ionization detectors or scintillators. The main problems that must be faced when using these devices for low background applications are:

- the contaminations from ${ }^{40} \mathrm{~K},{ }^{232} \mathrm{Th},{ }^{238} \mathrm{U}$ and their daughters;

- cosmogenic activation;

- selection and monitoring of all the materials and tools involved in the crystal growth;

- selection and monitoring of all the materials and tools used for crystal machining and storage;

One of the most known (and maybe optimized) crystals is Sodium Iodide (NaI), which finds many applications especially in the field of dark matter searches. The DAMA/LIBRA experiment used $\mathrm{NaI}(\mathrm{Tl})$ to search dark matter interactions in the form of nuclear recoils [1]. Dark matter particles in the galactic halo should produce a modulation in the rate of recoils, with period and phase compatible with the annual cycle. This experiment found a modulation signal with high statistical significance, that was so important to motivate the proposal of many other experiments aiming to probe the same signal with similar or different detection techniques.

The projects that decided to exploit the same crystals, however, soon discovered that growing radio-pure $\mathrm{NaI}$ is very challenging. The KIMs collaboration tried to grow crystals using NaI powders from different producers. The best result showed contaminations in ${ }^{40} \mathrm{~K}$ (a dangerous isotope for the background of dark matter searches) 2 times larger with respect to those of DAMA [2], likely because of the poor radio-purity of the starting powder. Similar results were obtained by the ANAIS collaboration, that was stuck on similar radioactive levels [3]. A more recent project, 
SABRE, proved that growing powders with high purity levels is feasible [4]. Nevertheless, the ${ }^{40} \mathrm{~K}$ contaminations in the first prototype crystal (Table 1) turned out to be around $30 \mathrm{ppb}$, again much larger than those of DAMA.

Table 1: Contaminations of the powders used by the DAMA experiment (first column) and of the powders produced by Seastar-MV Lab (second column) and Sigma Aldrich Astrograde (third column) in collaboration with SABRE. In the last column, contaminations in the final DAMA crystals are reported. Values marked with $(*)$ are preliminary. Data from [4].

DAMA Seastar-MV Lab Sigma Aldrich Astrograde DAMA CRYSTALS

\begin{tabular}{lcccc} 
& {$[\mathrm{ppb}]$} & {$[\mathrm{ppb}]$} & {$[\mathrm{ppb}]$} & {$[\mathrm{ppb}]$} \\
\hline $\mathrm{K}$ & 100 & 12 & $3.5-18$ & 13 \\
$\mathrm{Rb}$ & $\mathrm{N} / \mathrm{A}$ & 14 & 0.2 & $<0.35$ \\
$\mathrm{U}$ & $\sim 0.02$ & $0.0035^{*}$ & $<0.001^{*}$ & $0.0005-0.0075$ \\
$\mathrm{Th}$ & $\sim 0.02$ & $<0.001^{*}$ & $<0.001^{*}$ & $0.0007-0.01$
\end{tabular}

The difficulty in suppressing the final radio-active content of the crystal led to a long research activity targeting all the possible sources of re-contaminations. At the 2015 scientific committee at Laboratori Nazionali del Gran Sasso, the SABRE collaboration presented a first crystal with ${ }^{40} \mathrm{~K}$ activity similar to the one of DAMA, proving that this study was successful. Nevertheless, this was a single prototype and more tests are needed to confirm the result.

An interesting example of successful production of radio-pure crystals concerns the tellurium dioxide $\left(\mathrm{TeO}_{2}\right)$ crystals used by the CUORE experiment [5]. To reach the impressive radio-purity target of the experiment $\left(<3 \times 10^{-13} \mathrm{~g} / \mathrm{g}\right.$ in $\left.{ }^{238} \mathrm{U},{ }^{232} \mathrm{Th}\right)$, more than 20 years of R\&D were necessary. The procedure for the growth of $\mathrm{TeO}_{2}$ foresees a series of steps that must be done starting from pure powders, in controlled environment, and with severe checks at every stage [6]. Each phase of production is monitored exploiting the most advanced screening technologies, like:

- Inductively Coupled Plasma - Mass Spectrometry (ICP-MS) for contaminations in ${ }^{238} \mathrm{U}$, ${ }^{232} \mathrm{Th}$ in $\mathrm{Te}$ and $\mathrm{TeO}_{2}$, as well as in consumables and reagents. ICP-MS allows to reach a sensitivity of $10^{-12} \mathrm{~g} / \mathrm{g}$ on these isotopes; a similar sensitivity can be achieved also also on $\mathrm{Pb}$ and $\mathrm{Bi}$, while it is limited to $10^{-6} \mathrm{~g} / \mathrm{g}$ on ${ }^{40} \mathrm{~K}$.

- High Purity-Ge (HP-Ge) spectroscopy. The HP-Ge used for the study of $\mathrm{TeO}_{2}$ are located in the underground LNGS and feature a very high intrinsic radio-purity. They can reach sensitivity of $10^{-12} \mathrm{~g} / \mathrm{g}$ with high mass of source ( $\mathrm{kg}$ ) and a long measurement time (months). It should be observed that, in contrast to ICP-MS, HP-Ge is very sensitive also to ${ }^{40} \mathrm{~K}$, and to out-of-equilibrium contaminants.

- Surface Barrier Detectors (SBD) for surface contaminations of specific materials ad monitoring of selected components (in particular for lapping cloths, packaging, and so on).

Finally, the CUORE collaboration performed a bolometric run of sample crystals. This test consist in operating the crystal itself as a calorimeter, by equipping it with a sensor that can read the temperature rises produced by interactions in the crystal lattice. Even if rather expensive (as it requires 
a cryogenic run at $\sim 10 \mathrm{mK}$ ), bolometers are among the most sensitive detectors. The measurement of the CUORE crystals showed no evidence for bulk contaminations, and allowed to set an upper limit of $<1.8 \times 10^{-14} \mathrm{~g} / \mathrm{g}$ in ${ }^{238} \mathrm{U}$ and $<5.5 \times 10^{-14} \mathrm{~g} / \mathrm{g}$ in ${ }^{232} \mathrm{Th}$. The excellent contamination levels reached with $\mathrm{TeO}_{2}$ crystals prove that the strict protocol for the crystal production is very effective.

\subsection{A few complications}

The excellent results obtained by several collaborations, as those described in the previous section, may be not enough for next generations experiments.

First of all, several next-generation projects, especially in the field of neutrino-less double beta decay searches, will need isotopically enriched crystals [7]. The isotopic enrichment is worrisome for several reasons. It can be very expensive, meaning that the material loss during crystal growth have to be minimized. This is in contrast with the request of better and better mono-crystalline structures, as the growth of a crystal with a "good" structure is usually performed via subsequent growths, in which only the best quality powder is kept. Furthermore radio and chemical purity can become a serious issue. This is a well known problem for collaborations that use (for example) Molibdenum-based crystals: tens/hundreds of ppm of transition metals, that are typical contaminant of enriched Mo, can spoil the properties of the final crystal. As shown by the LUMINEU collaboration, such problems can be solved, but with extra R\&D effort [8].

Finally, maintaining the achieved purity is not simple: we need very strict protocols in order to avoid Radon implantation or re-contamination due to human activities. As an example, the CUORE crystals could come in contact only with ultra clean gloves, ultra pure water, polishing slurry, polishing pads, cleaned and conditioned polyethylene sheets and vacuum plastic bag, and all these materials had to be previously tested. At the end of operations, that had to be performed in a dedicated clean-room, crystals were packed in a triple vacuum package and stored by groups of six crystals in polyethylene vacuum boxes. The boxes were stored underground to prevent cosmic activation, which is a problem for many detectors.

These considerations motivate the recent proposal of developing dedicated underground growth facilities, which may be mandatory for next generation experiments.

\section{Copper}

Because of its natural radio-purity, as well as its excellent mechanical and cryogenic features, copper is the material of choice for the structural parts of different experiments. Copper can be produced in many different ways, according to the requirements on radio-purity, and on the content of "dangerous" elements such as oxygen and hydrogen. One of the copper that has been extensively characterized is the ETP1 (Electronic Tough Pitch, also called NOSV) copper used by experiments like CUORE. This material is poured in forms of ingots by melting electrolytically refined copper, whose chemical composition must ensure purity higher than $99.9 \%$ and low oxygen/ nitrogen content. Ingots are then rolled to improve the homogeneity of the grain structure, optimizing the mechanical properties. The obtained copper is eventually machined to obtain the final elements of the CUORE detector. 
Neutron Activation Analysis and HP-Ge spectroscopy showed no evidence of bulk contamination for this material, and allowed to set an upper limit on the activity of ${ }^{232} \mathrm{Th}$ and ${ }^{238} \mathrm{U}$ of $<2$ and $<70 \mu \mathrm{Bq} / \mathrm{kg}$ respectively.

The study of surface contaminations is always a delicate issue, as the achievement of a high sensitivity requires large surfaces and a long measurement time. As an example, to compare three different protocols for surface cleaning, the CUORE collaboration had to perform a bolometric run of several months, called the Three Towers Test [9]. Each cleaning procedure was tested by facing the treated copper to a detector made of twelve $5 \times 5 \times 5 \mathrm{~cm}^{3} \mathrm{TeO}_{2}$ crystals operated as bolometers. The large active surface, the absence of dead layers, the intrinsic radio-purity and the high energy resolution of bolometers, allowed to reach a sensitivity better than $10^{-7}-10^{-8} \mathrm{~Bq} / \mathrm{cm}^{2}$ on ${ }^{232} \mathrm{Th}$ and ${ }^{238} \mathrm{U}$. I highlight that bolometers could be used to test also materials other than copper with similar sensitivities.

Despite the excellent results achieved with NOSV copper, several next-generation experiment will need even more radio-pure materials. This is a problem also for the assay, as the detection techniques described up to now can not achieve the sufficient sensitivity. Thus, in many cases, only the final detector will be able to measure the actual contamination of the materials. Nevertheless, in this contribution I present two promising technologies that have been developed for the study of bulk and surface contaminations.

Concerning bulk contaminations, it is worthy mentioning the studies carried out with preconcentration of samples for ICP-MS [10]. This detection technique is based on concentrating the analytes while reducing the matrix elements in the final solution. The sample (in this case copper) is put into vials with the addition of some tracers, which allow to monitor the sample dilution. Than, high-concentration nitric acid is used to dissolve the sample, and in this process ${ }^{232} \mathrm{Th}$ and ${ }^{238} \mathrm{U}$ form a negatively-charged compound. The sample is passed through anion-exchange column; since the resin in the column is positively charged, it will capture ${ }^{232} \mathrm{Th}$ and ${ }^{238} \mathrm{U}$. The final eluent is analyzed using ICP-MS.

This technique allowed to reach impressive Method Detection Limits (MDL) of 0.034 and $0.131 \mu \mathrm{Bq} / \mathrm{kg}$ on ${ }^{232} \mathrm{Th}$ and ${ }^{238} \mathrm{U}$, respectively [10]. Apart from the excellent sensitivity, it brings the advantage of being relevant also for other materials: Lead $\left(0.4\right.$ and $17 \mu \mathrm{Bq} / \mathrm{kg}$ on ${ }^{232} \mathrm{Th}$ and ${ }^{238} \mathrm{U}$ respectively), Titanium ( 6 and $30 \mu \mathrm{Bq} / \mathrm{kg}$ on ${ }^{232} \mathrm{Th}$ and ${ }^{238} \mathrm{U}$ respectively), Stainless Steel (4 and $62 \mu \mathrm{Bq} / \mathrm{kg}$ on ${ }^{232} \mathrm{Th}$ and ${ }^{238} \mathrm{U}$ respectively) [11]. Nevertheless, it should be highlighted that ICP-MS can not assess isotopes that are out of the secular equilibrium, which is a limiting factor for many experiments.

Applying this technique also to the NOSV copper could allow to determine if this material is pure enough for next-generation experiments, but such measurements have not been performed yet. On the contrary, the material that was characterized with such a high sensitivity, and turned out to be so radio-pure, is the electro-formed copper. The idea at the basis of this production process is that, when electro-plating copper from a solution onto cathodes, most of the contaminants do not follow copper by virtue of their different electrochemical values. OFHC copper, which has an excellent starting radio-purity, is electro-deposited on on stainless steel forms that were previously machined to assume the shape of the final element. This process, that is done underground to reduce the activation of copper, resulted in an excellent purity of $0.3 \mu \mathrm{Bq} / \mathrm{kg}$ on both ${ }^{232} \mathrm{Th}$ and ${ }^{238} \mathrm{U}$. 
The missing element for a full characterization of electro-formed copper is the high-sensitivity study of its surface contaminations. The experience with NOSV copper proved that the production (and in particular the machining) of this material can introduce contaminations that, despite the cleaning procedure (realized with an aggressive sequence of tumbling, electropolishing, chemical etching and magnetron plasma etching), are of the level of tens of $\mathrm{nBq} / \mathrm{cm}^{2}$.

Achieving a similar sensitivity also on electro-formed copper is feasible using scintillating bolometers [12]. These detectors have several advantages:

- they can perform simultaneously $\alpha$ and $\gamma$ spectroscopy;

- they provide a wide active surface of hundreds of $\mathrm{cm}^{2}$;

- there are no dead layers;

- the energy resolution can be of the order of $0.1 \%$ over a wide energy range (from a few $\mathrm{keV}$ to a few $\mathrm{MeV}$ );

- they have a low intrinsic background.

Thanks to these features, we can reach a sensitivity of tens of $\mathrm{nBq} / \mathrm{cm}^{2}$ in a few weeks. Again, it is worthy saying that this technology can be applied to a wide range of materials.

\section{Conclusions}

The procurement of radio-pure materials is one of the main issues for different experiment aiming at low background. Furthermore, the required contamination levels are so low, that "standard" assay techniques can not assess them. In this contribution I presented the problems related to commonly used active and passive materials, crystals and copper. It is likely that both these materials will require dedicated underground production facilities to comply with the requirements of next generation experiments. Finally, I showed that the scientific community is proposing more and more sensitive assays for the investigation of such low contaminations. These newly-developed techniques can be applied to many commonly used material, and thus can be of great interest for several experiments.

\section{References}

[1] R. Bernabei, et al., "The Annual Modulation Signature for Dark Matter: DAMA/LIBRA-Phase1 Results and Perspectives", Adv. in High Energy Phys.2014 605659 (2014);

[2] Hyun Su Lee, "Development of low background CsI(Tl) and NaI(Tl) crystals for WIMP search", AIP Conf. Proc. 1672040002 (2015);

[3] J. Amarè et al., "Background analysis and status of the ANAIS dark matter project", AIP Conf. Proc. 1672100001 (2015);

[4] J. Xu, "SABRE? A test of DAMA with high-purity NaI(Tl) crystals", AIP Conf. Proc. 1672040001 (2015);

[5] CUORE Collaboration, "CUORE: A Cryogenic underground observatory for rare events", Nucl. Instrum. Meth. A 518 775-798 (2004); 
[6] C. Arnaboldi et al., "Production of high purity $\mathrm{TeO}_{2}$ single crystals for the study of neutrinoless double beta decay", J.Cryst.Growth 312 2999-3008 (2010);

[7] J.W. Beeman, "Double-beta decay investigation with highly pure enriched ${ }^{82}$ Se for the LUCIFER experiment", Eur. Phys. J. C 75591 (2015);

[8] V.N. Shlegel et al. "Purification of molybdenum oxide, growth and characterization of medium size zinc molybdate crystals for the LUMINEU program", EPJ Web of Conferences 6503001 (2014);

[9] F. Alessandria et al., "Validation of techniques to mitigate copper surface contamination in CUORE", Atropart. Phys. 45 13-22 (2013);

[10] B.D. La Ferriere et al.," A novel assay method for the trace determination of Th and U in copper and lead using inductively coupled plasma mass spectrometry", Nucl.Instrum.Meth. A 775 93-98 (2014);

[11] E. Hoppe?s talk, Low Radioactivity Techniques workshop 2015;

[12] L. Cardani et al., "A BGO scintillating bolometer for $\gamma$ and $\alpha$ spectroscopy", JINST 7 P10022 (2012); 